International Journal of Engineering \& Technology, $7(4.11)(2018) 276-280$
International Journal of Engineering \& Technology
SPC
Website: www.sciencepubco.com/index.php/IJET
Research paper

\title{
Profiling of Myocardial Infarction History from Electrocardiogram using Artificial Neural Network
}

\author{
Amerah Hanis Hussin, Ahmad Syukri Abdul Aziz, Megat Syahirul Amin Megat Ali* \\ Faculty of Electrical Engineering, Universiti Teknologi MARA, 40450 Shah Alam, Selangor, Malaysia \\ *Corresponding author E-mail: megatsyahirul@salam.uitm.edu.my
}

\begin{abstract}
Myocardial infarction is an irreversible damage of heart muscle caused by prolonged oxygen deficiency. As a result, the presence of damaged tissue will alter the normal sinus rhythm. Hence, the paper proposes to profile history of myocardial infarction from electrocardiogram using artificial neural network. Data for anterior and inferior myocardial infarction, as well as healthy control is acquired from PTB Diagnostic ECG Database. Subsequently, QRS power ratio features for different frequency zones are extracted from the preprocessed electrocardiogram. Discriminative ability of the features is assessed using k-nearest neighbor. The best combination of features with $99.7 \%$ testing accuracy is the power ratio composite that combines both low-frequency and mid-frequency information. An intelligent profiling model is successfully developed using the composite features and an optimized artificial neural network. The model was able to identify between different electrocardiogram groups with overall accuracy of $98.4 \%$ and mean squared error of less than 0.1 . Conclusively, the proposed signal processing approach has provided an improved alternative to the established methods from literature.
\end{abstract}

Keywords: Myocardial Infarction; ECG, Power Ratio; $k$-Nearest Neighbor; Artificial Neural Network.

\section{Introduction}

Electrocardiogram (ECG) is non-invasive electrical recording of the heart. The bio-potential signal arises from propagation of ionic impulses throughout the cardiac conduction system. These can be detected using bio-potential electrodes attached to the arms and legs to form the limb lead systems. The bipolar configuration measures the potential difference between different pairs of electrodes. Meanwhile, the augmented limbs are unipolar configurations with common reference derived from Goldberger's central terminal. The bipolar and augmented limb leads represents the frontal view of the heart. Implementation of multiple lead systems allows for localization of defects within the electrical conduction pathways [1]. The abnormalities manifest as deviations from normal sinus rhythms. Among the widely studied arrhythmias include premature ventricular contractions [2], bundle branch blocks [3], cardiomyopathy [4] and myocardial infarction (MI) [5].

Acute MI is the necrosis of heart tissue caused by prolonged ischemic conditions. Delay in treatment often results in cardiac arrest and death [6]. In the past, there has been an attempt to investigate the ECG of patients who survived acute MI. This was based on the assumption that the damaged myocardium would introduce irregularities to the sinus rhythm. The study focused on the power ratio features from the bipolar and augmented limb leads. Albeit the limited sample size, initial findings shows that the k-nearest neighbor model was able to classify between healthy ECG with those of anterior and interior MI survivors [7]. In this study however, a more thorough investigation is proposed by focusing on different frequency zones of the QRS complex; lowfrequency $(5-15 \mathrm{~Hz})$, mid-frequency $(15-80 \mathrm{~Hz})$ and highfrequency $(150-250 \mathrm{~Hz})$ components [5].

The discriminative ability of the proposed features can be assessed using k-nearest neighbor $(\mathrm{kNN})$. The technique relies on statistical principles in which instances from testing dataset are classified based on the largest occurrence of similarly labelled instances from the training dataset [8]. Meanwhile, the more advanced artificial neural network (ANN) is derived from biological functioning of neurons. The method is advantageous as it is capable of learning and can generalize solutions to a given problem [9]. Thus far, both kNN [10,11] and ANN [12, 13] have been widely used to classify features and model complex non-linear relationships for various biomedical applications.

By incorporating signal processing and intelligent modelling techniques, the study evaluates the feasibility of QRS power ratio features in different frequency zones for profiling ECG with history of MI. The investigation also compares the discriminative ability of the proposed features to that of the previous experiments [7] using larger sample size. The preceding analyses however, defined the behavior of QRS complex within a single frequency zone (1$20 \mathrm{~Hz})[14]$.

\section{Methodology}

Essentially, the work is divided into three major experiments. The first part of the study involves data collection, pre-processing and extraction of QRS power ratio features for low-frequency (LFQRS), mid-frequency (MF-QRS) and high-frequency (HF-QRS) components. The features are then segregated into anterior MI, inferior MI and healthy samples based on the medical records. Subsequently, the discriminative ability of proposed features is analysed using kNN. Apart from individually assessing the performance of bipolar and augmented limb leads, the work also includes feature composites in which various combination of frequency components are tested to enhance discrimination between the control groups. The best combination of features is then im- 
plemented in the profiling of MI history using ANN. Figure 1 shows the general framework of research methods.

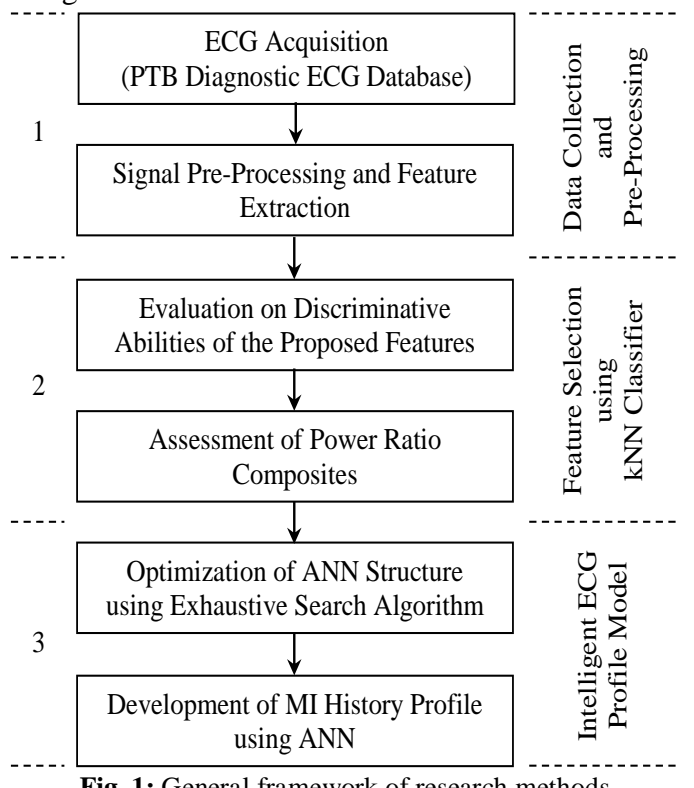

Fig. 1: General framework of research methods.

\subsection{Data collection}

Raw signal is obtained from PTB Diagnostic ECG Database. The ECG is acquired using non-commercial, Physikalisch-Technische Bundesanstalt prototype recorder with sampling rate of $1 \mathrm{kHz}$ [15]. Data is acquired for ECG with history of anterior MI and inferior MI, as well as healthy control.

\subsection{Pre-processing and feature extraction}

Signal pre-processing and extraction of QRS power ratio features are performed in MATLAB. The ECG is filtered into three frequency zones using band-pass finite impulse response (FIR) filters; LF-QRS (5-15 Hz), MF-QRS (15-80 Hz) and HF-QRS (150-250 $\mathrm{Hz}$ ). FIR filters are adopted as it is inherently stable and have linear phase response [16]. Subsequently, the pre-processed signal is segregated into smaller 5 seconds segments. The signal is then converted to power spectral density (PSD) using Welch method with 50\% overlapping epoch and length of 1024 [7].

Energy spectral density (ESD) is computed as the area under PSD curve. Information for bipolar limb leads is then normalized using in (1), (2) and (3). Notations I, II and III each represent information for Lead I, Lead II and Lead III.

$$
\begin{aligned}
& \text { PowerRatioI }=\frac{\mathrm{ESD}_{\mathrm{I}}}{\mathrm{ESD}_{\mathrm{I}}+\mathrm{ESD}_{\mathrm{II}}+\mathrm{ESD}_{\mathrm{III}}} . \\
& \text { PowerRatioII }=\frac{\mathrm{ESD}_{\mathrm{II}}}{\mathrm{ESD}_{\mathrm{I}}+\mathrm{ESD}_{\mathrm{II}}+\mathrm{ESD}_{\mathrm{III}}} . \\
& \text { PowerRatioIII }=\frac{\mathrm{ESD}_{\mathrm{III}}}{\mathrm{ESD}_{\mathrm{I}}+\mathrm{ESD}_{\mathrm{II}}+\mathrm{ESD}_{\mathrm{III}}} .
\end{aligned}
$$

Similarly, the information for augmented limb leads are each normalized through in (4), (5) and (6). Notations aVR, aVL and aVF each represents information for Lead aVR, Lead aVL and Lead aVF.

$$
\text { PowerRatioaVR }=\frac{\mathrm{ESD}_{\mathrm{aVR}}}{\mathrm{ESD}_{\mathrm{aVR}}+\mathrm{ESD}_{\mathrm{aVL}}+\mathrm{ESD}_{\mathrm{aVF}}}
$$

$$
\begin{gathered}
\text { PowerRatioaVL }=\frac{\mathrm{ESD}_{\mathrm{aVL}}}{\mathrm{ESD}_{\mathrm{aVR}}+\mathrm{ESD}_{\mathrm{aVL}}+\mathrm{ESD}_{\mathrm{aVF}}} . \\
\text { PowerRatioaVF }=\frac{\mathrm{ESD}_{\mathrm{aVF}}}{\mathrm{ESD}_{\mathrm{aVR}}+\mathrm{ESD}_{\mathrm{aVL}}+\mathrm{ESD}_{\mathrm{aVF}}} .
\end{gathered}
$$

The extracted features are subsequently clustered into the anterior MI, inferior MI and healthy control. Table 1 shows the individual ECG cluster and their respective indexes. The labels are required by both $\mathrm{kNN}$ and ANN for modelling purposes.

Table 1: ECG Clusters and the Assigned Index Labels

\begin{tabular}{|c|c|}
\hline Cluster & Index \\
\hline Anterior MI & 1 \\
\hline Inferior MI & 2 \\
\hline Healthy & 3 \\
\hline
\end{tabular}

A separate set of features based on methods adopted in the preceding experiment has been replicated. The analysis aims to observe the effect of increased sample size on the discriminative ability of the previously proposed features [7].

\subsection{Feature selection using $\mathrm{kNN}$}

Classification of features using $\mathrm{kNN}$ algorithm is relatively unsophisticated. The arrangement of data is initially randomized. $80 \%$ of the data is used for training and the remaining $20 \%$ for testing. The algorithm initially memorizes training features based on the corresponding ECG clusters. Consequently, the unlabelled features from the testing set are identified by assigning the most frequency class labels with $\mathrm{k}$ nearest training samples. Euclidean distance metric is adopted in this study and classification is performed for $\mathrm{k}=1$ to $\mathrm{k}=5$ [7].

Discriminative ability of the features is assessed in terms of accuracy (Acc), positive predictivity $(\mathrm{Pp})$ and sensitivity $(\mathrm{Se})$. Each of the parameters is expressed in (7), (8) and (9). TP, TN, FP and FN each represent true positive, true negative, false positive and false negative classifications.

$$
\begin{aligned}
& \mathrm{Acc}=\frac{\mathrm{TP}+\mathrm{TN}}{\mathrm{TP}+\mathrm{TN}+\mathrm{FP}+\mathrm{FN}} \times 100 \% . \\
& \mathrm{Pp}=\frac{\mathrm{TP}}{\mathrm{TP}+\mathrm{FP}} \times 100 \% . \\
& \mathrm{Se}=\frac{\mathrm{TP}}{\mathrm{TP}+\mathrm{FN}} \times 100 \% .
\end{aligned}
$$

To avoid bias issues, true performance is assessed using k-fold cross-validation technique. The cross-validation estimate is the total correct classification that is averaged over the number of folds within the dataset. Therefore, features are considered stable for specific dataset if comparable predictions are being made with different sets of testing features [17].

The dataset is randomly divided into five disjointed folds. At each instant, four folds of feature sets are used for training and the remaining fold is used for testing. These correlates to the 80:20 split ratio that has been set for kNN classification. At varying iterations of $\mathrm{k}$, different combination of folds will form dissimilar training and testing sets. The true classification performance is therefore averaged over five instances of $\mathrm{k}$.

To compare effectiveness of the proposed features for discriminating between healthy control and those with history of anterior MI and inferior MI, the initial assessment is performed separately for bipolar and augmented limb leads. An extended study is also conducted on feature composites that combine different frequency zones of the QRS complex. The best power ratio composites will be used to develop an intelligent profiling model using ANN. 


\subsection{Intelligent ECG profiling model using ANN}

ANN is generally comprised of an input layer, single hidden layer and an output layer. The input is comprised of selected QRS power ratio features. Meanwhile, the output corresponds to the index labels of the ECG clusters. The study implements tangent sigmoid as activation function for the hidden layer. Meanwhile, the output neuron adopts pure linear function. Network training is performed using the Levenberg-Marquardt algorithm. Training, testing and validation dataset is randomly segregated with 70:15:15 split ratio. The optimum number of hidden neurons is assessed through exhaustive search algorithm.

The algorithm generally implements a constructive approach [18], while considering the rules of thumb for selecting the minimum and maximum number of hidden neurons. The lower limit is selected as $2 / 3$ the number of input and output neurons, whereas the maximum threshold is less than twice the number of input neurons [19]. The algorithm implements the same set of features to train the network with different hidden neuron settings. As shown in Figure 2, training starts with minimum hidden neurons. For each of the hidden neuron settings, the process is repeated for 40 iterations. Subsequently, the process restarts with increased number of hidden neurons until the maximum limit is reached.

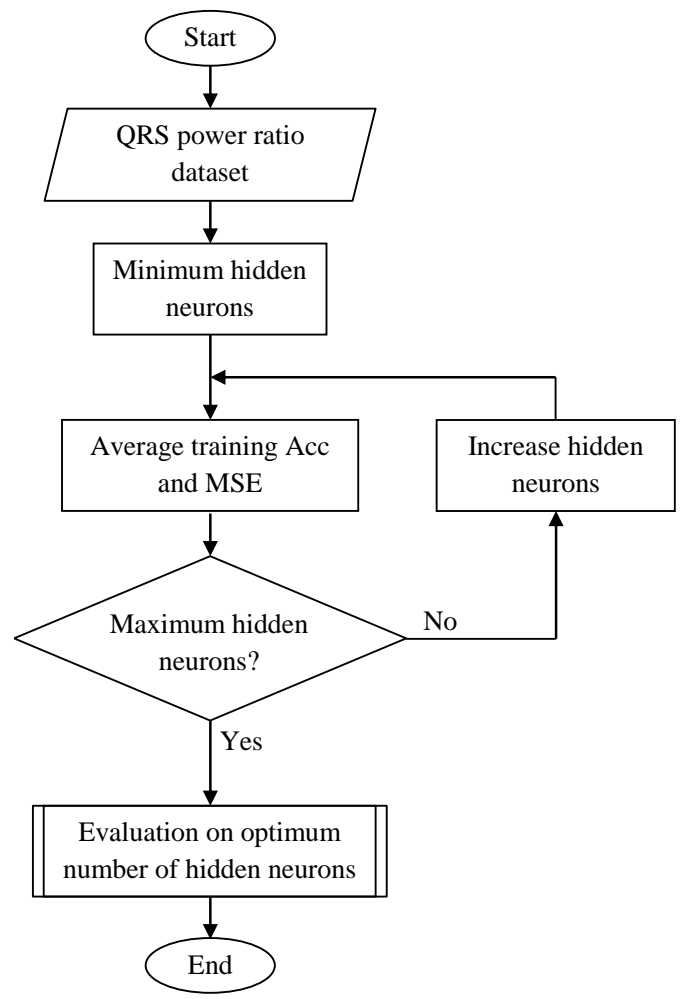

Fig. 2: Exhaustive search algorithm. MSE = Mean Squared Error.

Subsequently, the optimum number of hidden neurons is selected based on the highest average training accuracy with lowest MSE [20]. The network is then finalized and trained to achieve the best classification. Similar parameters on Acc, $\mathrm{Pp}$ and $\mathrm{Se}$ are used to assess model performance.

\section{Results and Discussion}

\subsection{Data segregation and feature extraction}

ECG with history of anterior and inferior MI, as well as healthy control was downloaded in .mat format. Table 2 compares the number of subjects involved in current work and those of the published literature. Data from a total of 87 subjects has been acquired for this study. This surpasses the preceding work that was based on 35 subjects [7].
Table 2: Comparison between Number of Subject in Current Work and Published Literature [7]

\begin{tabular}{|c|c|c|}
\hline Group & Current Work & Published Literature \\
\hline Anterior MI & 27 & 8 \\
\hline Inferior MI & 36 & 7 \\
\hline Healthy & 24 & 20 \\
\hline
\end{tabular}

The raw ECG was pre-processed for noise removal. Subsequently, the signal is then filtered into the respective frequency zones before being segregated into 5 seconds signal segments. The feature extraction stage yielded 1206 samples for each ECG class.

\subsection{Discriminative ability of power ratio features}

Initially, the study performs separate analysis on bipolar and augmented limb leads for LF-QRS, MF-QRS and HF-QRS components. In addition, the approach in published literature has been replicated with larger sample size and performance of the features is observed. As summarized in Table 3, the performance between bipolar and augmented limb leads is comparable with LF-QRS yielding the highest testing accuracy. Slight reduction in performance has been observed for MF-QRS component. The very low testing accuracy for HF-QRS indicates its poor discriminative ability. None of the proposed set of features exceeded testing accuracy of $90.0 \%$. An extended analysis with previous study shows comparable results. These are attributed to the overlapping frequency ranges of the proposed 5-15 Hz for LF-QRS and 1-20 Hz from the established method [7].

Table 3: Five-Fold Average Accuracy for Power Ratio of Bipolar and Augmented Limb Leads $(\mathrm{k}=2)$

\begin{tabular}{|c|c|c|c|c|}
\hline \multirow{2}{*}{ Power Ratio } & \multicolumn{2}{|c|}{ Bipolar Limb Leads } & \multicolumn{2}{c|}{ Augmented Limb Leads } \\
\cline { 2 - 5 } & Training & Testing & Training & Testing \\
\hline Literature [7] & $100.0 \%$ & $87.5 \%$ & $100.0 \%$ & $87.3 \%$ \\
\hline LF-QRS & $100.0 \%$ & $87.6 \%$ & $100.0 \%$ & $88.3 \%$ \\
\hline MF-QRS & $100.0 \%$ & $86.6 \%$ & $100.0 \%$ & $86.8 \%$ \\
\hline HF-QRS & $100.0 \%$ & $45.5 \%$ & $100.0 \%$ & $46.5 \%$ \\
\hline
\end{tabular}

The work then shifts its focus on a different aspect by combining features from bipolar and augmented limb leads for the respective frequency zones. Even with increase in the number of features, the method was not able to significantly improve the testing accuracy. Hence, a study that focuses on composite features is required.

Table 3: Five-Fold Average Accuracy for LF-QRS, MF-QRS and HFQRS components $(\mathrm{k}=2)$

\begin{tabular}{|c|c|c|c|}
\hline Power Ratio & LF-QRS & MF-QRS & HF-QRS \\
\hline Training & $100.0 \%$ & $100.0 \%$ & $100.0 \%$ \\
\hline Testing & $88.2 \%$ & $86.6 \%$ & $46.4 \%$ \\
\hline
\end{tabular}

\subsection{Power ratio composites}

In an attempt to improve the discriminative ability of QRS power ratio features, combination between different frequency zones was evaluated. The study focused on the following composites; lowfrequency and mid-frequency (LF-MF), low-frequency and highfrequency (LF-HF), as well as mid-frequency and high-frequency (MF-HF) combinations. The composites were tested separately for bipolar and augmented limb leads. Results in Table 4 indicate marked improvement on testing accuracy compared to the preceding experiments.

Table 4: Five-Fold Average Accuracy for Power Ratio Composites of Bipolar and Augmented Limb Leads $(\mathrm{k}=2)$

\begin{tabular}{|c|c|c|c|c|}
\hline \multirow{2}{*}{$\begin{array}{c}\text { Power Ratio } \\
\text { Composites }\end{array}$} & \multicolumn{2}{|c|}{ Bipolar Limb Leads } & \multicolumn{2}{c|}{ Augmented Limb Leads } \\
\cline { 2 - 5 } & Training & Testing & Training & Testing \\
\hline LF-MF & $100.0 \%$ & $99.7 \%$ & $100.0 \%$ & $99.7 \%$ \\
\hline LF-HF & $100.0 \%$ & $96.7 \%$ & $100.0 \%$ & $96.4 \%$ \\
\hline MF-HF & $100.0 \%$ & $96.5 \%$ & $100.0 \%$ & $96.5 \%$ \\
\hline
\end{tabular}

Generally, results between bipolar and augmented limb leads are comparable with LF-MF power ratio composite yielding the highest testing accuracy of close to $100.0 \%$.

Table 5 shows the Pp and Se measures that were obtained for both bipolar and augmented limb leads. Findings indicate marked improvement on the discriminative ability of the new feature compo- 
site. As both bipolar and augmented limb lead components yielded comparable results, only one will be used in the intelligent profiling model.

Table 5: Five-Fold Average Pp and Se for LF-MF Power Ratio Composite $(\mathrm{k}=2)$

\begin{tabular}{|c|c|c|c|c|}
\hline \multirow{2}{*}{$\begin{array}{c}\text { LF-MF Power } \\
\text { Ratio Composites }\end{array}$} & \multicolumn{2}{|c|}{ Bipolar Limb Leads } & \multicolumn{2}{c|}{ Augmented Limb Leads } \\
\cline { 2 - 5 } & $\mathrm{Pp}$ & $\mathrm{Se}$ & $\mathrm{Pp}$ & $\mathrm{Se}$ \\
\hline Anterior MI & $99.6 \%$ & $99.6 \%$ & $99.7 \%$ & $99.6 \%$ \\
\hline Inferior MI & $99.8 \%$ & $99.6 \%$ & $99.8 \%$ & $99.7 \%$ \\
\hline Healthy & $99.7 \%$ & $99.8 \%$ & $99.7 \%$ & $99.8 \%$ \\
\hline
\end{tabular}

By observing the resultant performance of different sets of power ratio features, it was evident that the proposed HF-QRS has the lowest discriminative capabilities. Findings were consistent with the combined feature settings where both LF-HF and MF-HF was not able to match the performance of LF-MF composites.

\subsection{Development of intelligent profiling model}

In the final part of the study, LF-MF power ratio composite for the bipolar limb leads and ANN was implemented to develop an intelligent ECG profile model. Initial, the structure is comprised of six input and one output neurons. The optimum number of hidden neurons was assessed through the exhaustive search algorithm. The minimum and maximum number of hidden neurons was each set at five and 11. The average training accuracies and MSE for are shown in Figure 3 and Figure 4. From the obtained results, the optimum number of hidden neurons for the final network structure is 11. This was selected based on the highest training accuracy with lowest MSE.

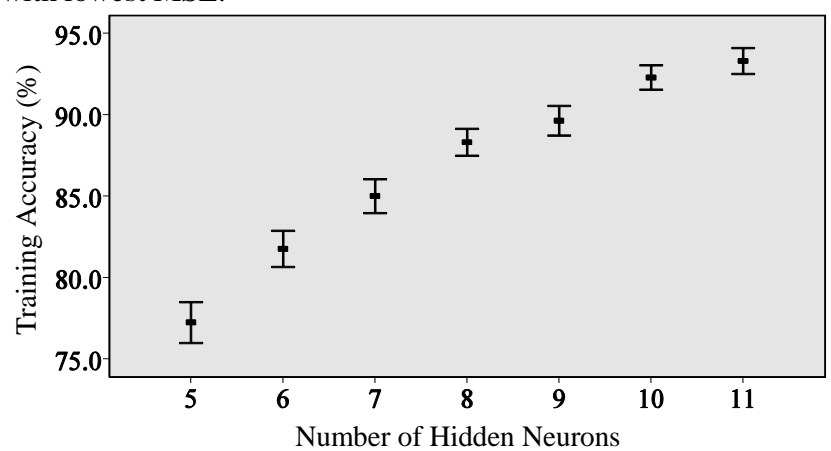

Fig. 3: Average training accuracy.

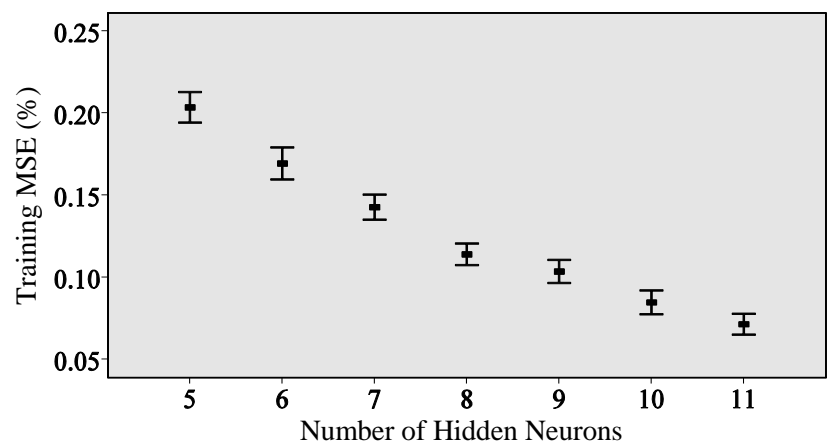

Fig. 4: Average training MSE.

The optimized network is then trained for best model performance. Table 6 shows the classification accuracies and MSE for training, validation and testing.

Table 6: Classification Performance for Intelligent Profiling Model

\begin{tabular}{|c|c|c|c|}
\hline Performance & Training & Validation & Testing \\
\hline Accuracy (\%) & $99.0 \%$ & $98.3 \%$ & $98.5 \%$ \\
\hline MSE & 0.0415 & 0.0491 & 0.0353 \\
\hline Correct Classifications & 2,540 & 509 & 512 \\
\hline
\end{tabular}

Generally, the network model has successfully classified the LFMF power ratio composites into the respective ECG clusters with overall accuracy of $98.4 \%$. The performance has been consistent throughout training, validation and testing stages and the obtained MSE is less than 0.1. Such performance is expected as kNN has demonstrated excellent discriminative capabilities of the selected feature composites.

\section{Conclusion}

The investigation has initially proposed QRS power ratio for profiling ECG with history of anterior and inferior MI. The features were segregated into different frequency components. Findings demonstrate that performance of low-frequency information is comparable to the selected method from the literature. When assessed separately, LF-QRS and MF-QRS component yielded satisfactory performance for both bipolar and augmented limb leads. HF-QRS however, has demonstrated poor discriminative ability. By combining information from LF-QRS and MF-QRS components, the discriminative ability of newly formed LF-MF power ratio composites is significantly improved. The selected set of features and ANN was successfully implemented in the development of intelligent profiling model. Generally, the optimized network was able to classify between ECG with history of anterior and inferior MI, as well as healthy control; yielding excellent accuracy.

\section{Acknowledgement}

The study is supported by Institute of Research Management and Innovation, Universiti Teknologi MARA through the LESTARI research grant (600-IRMI/MyRA 5/3/LESTARI (015/2017)).

\section{References}

[1] Thygesen K, Alpert JS \& White HD (2007), Universal definition of myocardial infarction. European Heart Journal 28, 2525-2538.

[2] Inan OT, Giovangrandi L \& Kovacs GTA (2006), Robust neuralnetwork-based classification of premature ventricular contractions using wavelet transform and timing interval features. IEEE Transactions on Biomedical Engineering 53, 2507-2515.

[3] Megat Ali MSA, Jahidin AH, Norali AN \& Mat Som MH (2011), Classification of bundle branch blocks using multilayered perceptron network. Proceedings of IEEE International Conference on Control System, Computing and Engineering, pp. 531-535.

[4] Ahmad Shukri MH, Megat Ali MSA, Noor MZH, Jahidin AH, Saaid MF \& Zolkapli M (2012), Investigation on Elman neural network for detection of cardiomyopathy. Proceedings of IEEE Control and System Graduate Research Colloquium, pp. 328-332.

[5] Tsutsumi T, Okamoto Y, Kubota-Takano N, Wakatsuki D, Suzuki H, Sezaki K, Iwasawa K \& Nakajima T (2014), Time-frequency analysis of the QRS complex in patients with ischemic cardiomyopathy and myocardial infarction. IJC Heart and Vessels 4, 177-187.

[6] Tunstall-Pedoe H, Kuulasmaa K, Amouyel P, Arveiler D, Rajakangas AM \& Pajak A (1994), Myocardial infarction and coronary deaths in the World Health Organization MONICA Project, Circulation 90, 583-612.

[7] Megat Ali MSA, Jahidin AH, Yassin IM, Pasya I, Abdul Khalid MF \& Awang Z (2017), Characterization of post-MI electrocardiogram using power ratio features and k-nearest neighbor classifier. Journal of Fundamental and Applied Sciences 9, 937-951.

[8] Megat Ali MSA, Jahidin AH, Md Tahir N \& Taib MN (2014), Learning style classification via EEG sub-band spectral centroid frequency features. International Journal of Electrical and Computer Engineering 4, 931-938.

[9] Megat Ali MSA, Che Zainal CZA, Husman A, Saaid MF, Noor MZH \& Jahidin AH (2012), Detection of cardiomyopathy using multilayered perceptron network. Proceedings of IEEE 8th International Colloquium on Signal Processing and its Applications, pp. 436-440.

[10] Guraliuc AR, Barsocchi P, Potorti F \& Nepa P (2011), Limb movements classification using wearable wireless transceivers. IEEE Transactions on Information Technology in Biomedicine 15 474-480.

[11] Haron MH, Taib MN, Megat Ali MSA \& Mohd Yunus M (2012), Gender classification based on human radiation frequencies of chakra points and brain regions. Proceedings of IEEE International Conference on Electronics Design, Systems and Applications, pp. $188-193$. 
[12] Jahidin AH, Taib MN, Md Tahir N, Megat Ali MSA, Yassin IM, Lias S, Mohd Isa R, Omar WRW \& Fuad N (2013). Classification of intelligence quotient using EEG sub-band power ratio and ANN during mental task. Proceedings of IEEE Conference on Systems, Process and Control, pp. 204-208.

[13] Amiruddin AI, Megat Ali MSA, Saaid MF, Jahidin AH \& Noor MZH (2013), Feature reduction and arrhythmia classification via hybrid multilayered perceptron network. Proceedings of IEEE 3rd International Conference on System Engineering and Technology, pp. 290-294.

[14] Lin C-H (2008), Frequency-domain features for ECG beat discrimination using grey relational analysis-based classifier. Computers and Mathematics with Applications 55, 680-690.

[15] Goldberger AL, Amaral LAN, Glass L, Hausdorff JM, Ivanov PCh, Mark RG, Mietus JE, Moody GBPeng C-K \& Stanley HE (2000) PhysioBank, PhysioToolkit, and PhysioNet: Components of a new research resource for complex physiologic signals. Circulation 101 e215-e220.

[16] Charalambous C (1985), The performance of an algorithm for minimax design of two-dimensional linear phase FIR digital filters. IEEE Transactions on Circuits and Systems 32, 1016-1028.

[17] Kohavi R (1995), A study of cross-validation and bootstrap for accuracy estimation and model selection. Proceedings of International Joint Conference on Artificial Intelligence, pp. 1137-1145.

[18] Islam MM \& Murase K (2001), A new algorithm to design compact two-hidden-layer artificial neural networks. Neural Network 14, 1265-1278.

[19] Xu S \& Chen L (2008), A novel approach for determining the optimal number of hidden layer neurons for FNN's and its application in data mining. Proceedings of 5th International Conference on Information Technology and Applications, pp. 683-686.

[20] Benardos PG \& Voniakos G-C (2007), Optimizing feedforward artificial neural network architecture. Engineering Applications of Artificial Intelligence 20, 365-382. 